\title{
AstroGrid-D: Grid technology for astronomical science
}

\author{
Harry Enke $^{\mathrm{a}, *}$, Matthias Steinmetz ${ }^{\mathrm{a}}$, Hans-Martin Adorf ${ }^{\mathrm{b}}$, Alexander Beck-Ratzka ${ }^{\mathrm{c}}$, Frank Breitling ${ }^{\mathrm{a}}$, \\ Thomas Brüsemeister $^{\mathrm{d}}$, Arthur Carlson ${ }^{\mathrm{f}}$, Torsten Ensslin ${ }^{\mathrm{b}}$, Mikael Högqvist ${ }^{\mathrm{e}}$, Iliya Nickelt ${ }^{\mathrm{a}}$, Thomas Radke $^{\mathrm{c}}$, \\ Alexander Reinefeld ${ }^{\mathrm{e}}$, Angelika Reiser ${ }^{\mathrm{g}}$, Tobias Scholl ${ }^{\mathrm{g}}$, Rainer Spurzem ${ }^{\mathrm{d}, \mathrm{h}}$, Jürgen Steinacker ${ }^{\mathrm{d}}$, \\ Wolfgang Voges ${ }^{\mathrm{f}}$, Joachim Wambsgan ${ }^{\mathrm{d}}{ }^{\text {, Steve White }}{ }^{\mathrm{a}}$
}

${ }^{a}$ Astrophysikalisches Institut Potsdam AIP, Potsdam, Germany

${ }^{\mathrm{b}}$ Max-Planck-Institut für Astrophysik MPA, Garching, Germany

${ }^{c}$ Max-Planck-Institut für Gravitationsphysik (Albert-Einstein Institut) AEI, Potsdam, Germany

${ }^{\mathrm{d}}$ Astronomisches Recheninstitut am Zentrum für Astronomie Heidelberg ZAH, Heidelberg, Germany

${ }^{\mathrm{e}}$ Konrad-Zuse-Zentrum für Informationstechnik Berlin ZIB, Berlin, Germany

${ }^{\mathrm{f}}$ Max-Planck-Institut für extraterrestrische Physik MPE, Garching, Germany

${ }^{\mathrm{g}}$ Technische Universität München, Institut für Informatik TUM, Garching, Germany

${ }^{\mathrm{h}}$ National Astronomical Observatories of China, Chinese Academy of Sciences NAOC/CAS, 20A Datun Rd., Chaoyang District, Beijing 100012, China

\section{A R T I C L E I N F O}

\section{Article history:}

Received 21 April 2010

Received in revised form 28 June 2010

Accepted 20 July 2010

Available online 29 July 2010

Communicated by J. Makino

\section{Keywords:}

Methods: data analysis

Methods: numerical

Techniques: image processing

Telescopes

\begin{abstract}
A B S T R A C T
We present status and results of AstroGrid-D, a joint effort of astrophysicists and computer scientists to employ grid technology for scientific applications. AstroGrid-D provides access to a network of distributed machines with a set of commands as well as software interfaces. It allows simple use of computer and storage facilities and to schedule or monitor compute tasks and data management. It is based on the Globus Toolkit middleware (GT4).

Chapter 1 describes the context which led to the demand for advanced software solutions in Astrophysics, and we state the goals of the project.

We then present characteristic astrophysical applications that have been implemented on AstroGrid-D in chapter 2. We describe simulations of different complexity, compute-intensive calculations running on multiple sites (Section 2.1), and advanced applications for specific scientific purposes (Section 2.2), such as a connection to robotic telescopes (Section 2.2.3). We can show from these examples how grid execution improves e.g. the scientific workflow.

Chapter 3 explains the software tools and services that we adapted or newly developed. Section 3.1 is focused on the administrative aspects of the infrastructure, to manage users and monitor activity. Section 3.2 characterises the central components of our architecture: The AstroGrid-D information service to collect and store metadata, a file management system, the data management system, and a job manager for automatic submission of compute tasks.

We summarise the successfully established infrastructure in chapter 4, concluding with our future plans to establish AstroGrid-D as a platform of modern e-Astronomy.
\end{abstract}

() 2010 Elsevier B.V. All rights reserved.

\section{Introduction}

Astrophysical research is an intent driver for advances in computer science, especially so for high performance computing and data intensive calculations. We are used to the continuous increase of processor power which increases the potential of computer based analysis. Even faster is the rise of sensor size and storage capacity, both of which in recent years have grown even stronger than Moore's Law would predict. Unfortunately, this trend of growing data volumes also increases the complexity of the data

\footnotetext{
* Corresponding author. Tel.: +49 0331 7499433; fax: +49 03317499429

E-mail address: henke@aip.de (H. Enke).
}

management, as well as the processing, analysis and visualisation. Above a certain level, new methods have to be applied, e.g. the management of data becomes a task that is no longer trivial enough for a file system alone. This challenge affects many other domains outside of Astrophysics in the same way, and it is an important challenge to find answers, since in several research areas further progress depends on the successful processing of data volumes in the high Terabyte or Petabyte scale.

One solution for improved data management is the recent success in meta data standardisation and advanced corresponding protocols. In astrophysics this approach has led to the international "Virtual Observatory" initiative, which now allows for a fast search within extensive volumes of diverse stored data. 
But computer science itself has also researched ways to improve infrastructure usage and simplify the processing of information. The most compelling answer of recent years was the massive development in Grid computing, where a new software layer is used to connect distributed information infrastructures like clusters, storage servers and desktops to a loose network (see Foster, 2008).

Several research grid infrastructures were successfully set up in the past years. The most impressive example is the US "TeraGrid", funded since 2001 by the National Science Foundation. It offers over a petaflop of total compute capabilities and many different services and gateways to thousands of US scientists. Like the Open Science Grid, TeraGrid is based on the Globus Toolkit, enlarged by an auxilary software package set.

The European enterprise EGEE ("Enabling Grids for E-SciencE") was started 2004 as a EU project, sponsored from the European Union's research framework. EGEE was at the beginning mostly driven by the CERN's new large Hadron Collider and its demand for compute power. It currently combines about 40.000 CPUs and will in 2010 be transferred into a new body called EGI (European Grid Initiative). It will then focus mostly on the role to coordinate the collaboration of the national grid initiatives with supported middlewares limited to gLite, UNICORE and ARC.

The German national Grid initiative was inaugurated in 2004 by the Federal Research ministry. It has seen two main stages: D-Grid 1 (2005-2008) focussed on Grid application for fundamental sciences, whereas D-Grid 2 (2007-2010) mostly researched Grid use in applied sciences and industry.

The AstroGrid-D project was part of the first D-Grid initiative and started in 2005. Five major German astronomy institutes participated: AIP, AEI, MPA, MPE, and ZAH, together with computer science groups from the ZIB Supercomputer center and TUM. They collaborated on the common project goal: To establish a collaborative working environment for astronomy which provides the users with the powerful and reliable software tools and allows easy access to compute and storage facilities for their scientific work.

To achieve this the projected aimed to:

- set up a grid-based infrastructure for astronomical and astrophysical research,

- embed existing computational facilities, astronomical software applications, data archives and instruments,

- integrate this grid infrastructure into the national D-Grid environment,

- provide support for other astronomical groups to join,

- strengthen international partnerships.

AstroGrid-D has reached these goals in its setup phase which ended early 2009. The most important results were the first Virtual Organisation management, now the D-Grid-standard (see Section 3.1.1), integration of special hardware D-Grid (Sections 2.1.2 and 2.2.3) and the production run of one of the most compute-intensive scientific grid application to date Section 2.1.3.

We hereby present our experiences and results in some detail. The paper is grouped into two main chapters: First the astrophysical applications Section 2 and secondly our developments in information technology Section 3. In the summary Section (4) we give an outlook on our future plans.

\section{Astronomy and grid: astronomical use cases running on the project network}

Most areas of Astronomical research can profit from e-Science concepts and grid technology in particular.

In the course of the project, a total of twenty selected astronomic pilot applications were modified for grid use and imple- mented. Use cases ranged from compute-intensive simulations running on clusters, task farming jobs to explore large parameter spaces, analyzing programs accessing astronomical databases, to complex and specific applications as described below. These use cases also served to define the requirements for AstroGrid-D components.

When considering a grid implementation for a given application, it is decisive to compare how time-consuming and complex the task will be compared to the benefits, such as speed gain. Before we describe examples in detail we will state general experiences for different application classes.

For large simulations, e.g. from cosmology (Mare Nostrum, WebLinks, 2010), a grid environment is ideal to reduce typical obstacles. In a grid infrastructure, a unified and standardised interface is provided to access the grid-enabled resources of a high performance computing center. The Grid offers a common way to execute calculations and manage resulting data. Also many details, such as efficient data transfer, are handled by the Grid middleware. The need to learn details about a specific center is minimised.

Taskfarming jobs benefit from the grid infrastructure since there now is a multitude of resources available to them, as shown for the Geo600-example (Section 2.1.3). Especially applications with limited requirements can gain immensely from a grid implementation, where many hundreds of instances can be executed concurrently.

Robotic telescopes (Section 2.2.3) serve as an example for special scientific hardware. When combined to a worldwide network on the basis of grid middleware, this brings important advantages to coordinated observations. Typical tasks for such a network are multi-wavelength campaigns or the continuous monitoring of transient astronomical objects. A grid-based network simplifies coordination and infrastructure management, since grid devices such as storage servers and databases are easy to connect. Moreover, global grid schedulers can automatically coordinate and optimise the observations.

For large data sets like the Sloan Digital Sky Survey (SDSS, WebLinks, 2010) or the Millenium simulation archive (Springel et al., 2005), efficient processing poses a huge problem. The data often have inconsistent formats and interfaces, and the methods still vary how to define subsets and correlate them, or even run algorithms against them. To select data, the scientist needs access to a given database and, in most cases, also access to additional data files. Corresponding results must be stored in some accessible device. Since the data volumes are growing large and the catalogues may be distributed, techniques for data discovery searching, and transmission (data streaming) are applied, combined with mechanisms for parallelisation and load-balancing for the computing processes. At this point, Grid data processing overcomes the limits of the centralised data processing approach where so far large volumes of data are transferred to the application that requests them. The alternative is to distribute the data processing within the grid and the use of storage facilities accessible via grid methods. Whenever possible the application is executed at the location of the data.

Many solutions and design decisions, such as described in the last paragraph, rely on the work and standards of the Virtual Observatory. Hence AstroGrid-D collaborates closely with the German Astrophysical Virtual Observatory (GAVO), for example when using GAVOs easy-to-use data access interface to $\mathrm{N}$-body simulations. Via GAVO's participation in the IVOA activities, AstroGrid-D also participated from the developments where grid middleware is used to provide VObs services. We will continue the collaboration between AstroGrid-D and GAVO in the creation of a virtual data center for astronomy.

To support users in the deployment of their application, we compiled an application-to-grid guide that illustrates the steps to grid-enable simple applications (App2Grid, WebLinks, 2010). 


\subsection{Compute-intensive generic applications}

Many compute-intensive applications can be subdivided into multiple small parallel tasks that can run independently, e.g. on multiple grid resources. This can usually be achieved by partitioning the physical properties of the relevant parameter space. In the following, we will discuss three such compute-intensive grid applications, namely the task farming use case Dynamo, NBDOY6++ as an example use case with little $\mathrm{I} / \mathrm{O}$, and the gravitation wave analysis tool GEO600.

We have found that a grid implementation for this application type can be very beneficial and achieved within a manageable timeframe.

\subsubsection{Dynamo}

The Dynamo package shows how to use the advantages of grid computing without complex programming. Grid implementation is achieved by a shell script, that is lean, relatively simple to understand and easy to configure. It provides a grid connection for the purpose of task farming of serial programs, i.e. the launching of many instances of scientific software where the input differs for each run. We call this type of application atomic, since as a serial calculation it requires no further communication until the results are produced.

The scientific problem for this example is derived from the field of Magneto-Hydro-Dynamics. Rotation and turbulence in stars, accretion disks, and galaxies produce a magnetic field by the dynamo effect. In the case shown here the numerical simulation solves the induction equation with a turbulent electromotive force (alpha tensor). The general parameter dependence as well as the time development of a given set are studied, with special focus on the "flip-flop"-phenomenon of star spots (see Elstner and Korhonen, 2005).

For grid task farming with varying input sets the script reads in any number of input directories, each of which contains different data. Together with the executable, the job is then submitted iter- atively to grid resources specified in a list and executed there. Intermediate output can be retrieved on the fly; a visualisation example is shown in Fig. 1.

This solution is currently being applied to a similar use case for GAVO. Upgrades of the software would properly include GridSphere and improve the stage-in process. The script package can be downloaded from the AstroGrid-D use case web pages (Dynamo, WebLinks, 2010). Users with a demand for atomic, serial jobs should find this solution easy to implement within AstroGrid-D or within similar, Globus-based grids.

\subsubsection{NBODY6++ and $\varphi$ GRAPE}

NBODY6++ and $\varphi$ GRAPE are two variants of a family of high-order accurate direct $N$-body simulation codes, which are built upon the development of a series of earlier versions (1-6) of NBODY codes (Aarseth, 1999). $\varphi$ GRAPE is the only parallel code of this type to use special purpose GRAPE6 hardware (Harfst et al., 2007) based on GRAPE which has been designed by the University of Tokyo to accelerate gravitational force computations between particles (Makino et al., 2003; Fukushige et al., 2005). While $\varphi$ GRAPE is just a plain direct parallel NBODY code using a 4th order Hermite integrator with hierarchical block time steps, NBODY6++ is a parallel version of NBODY6 (with regularisation of close encounters, Ahmad-Cohen neighbour scheme, and other features), which is optimised for parallel general purpose supercomputers (Spurzem, 1999).

Examples for applications where gravitational forces between many bodies have to be calculated are globular clusters, young forming star clusters or central dense star clusters in galactic nuclei. Recent typical research using direct $N$-body simulations includes, e.g. models of galactic star clusters with many binaries (Hurley et al., 2007) or massive binary black holes embedded in dense stellar systems leading to coalescence and gravitational wave emission (Berczik et al., 2005, 2006; Berentzen et al., 2009).

NBODY6++ and $\varphi$ GRAPE are use cases of AstroGrid-D which supports deployment and execution of these as jobs on its re-
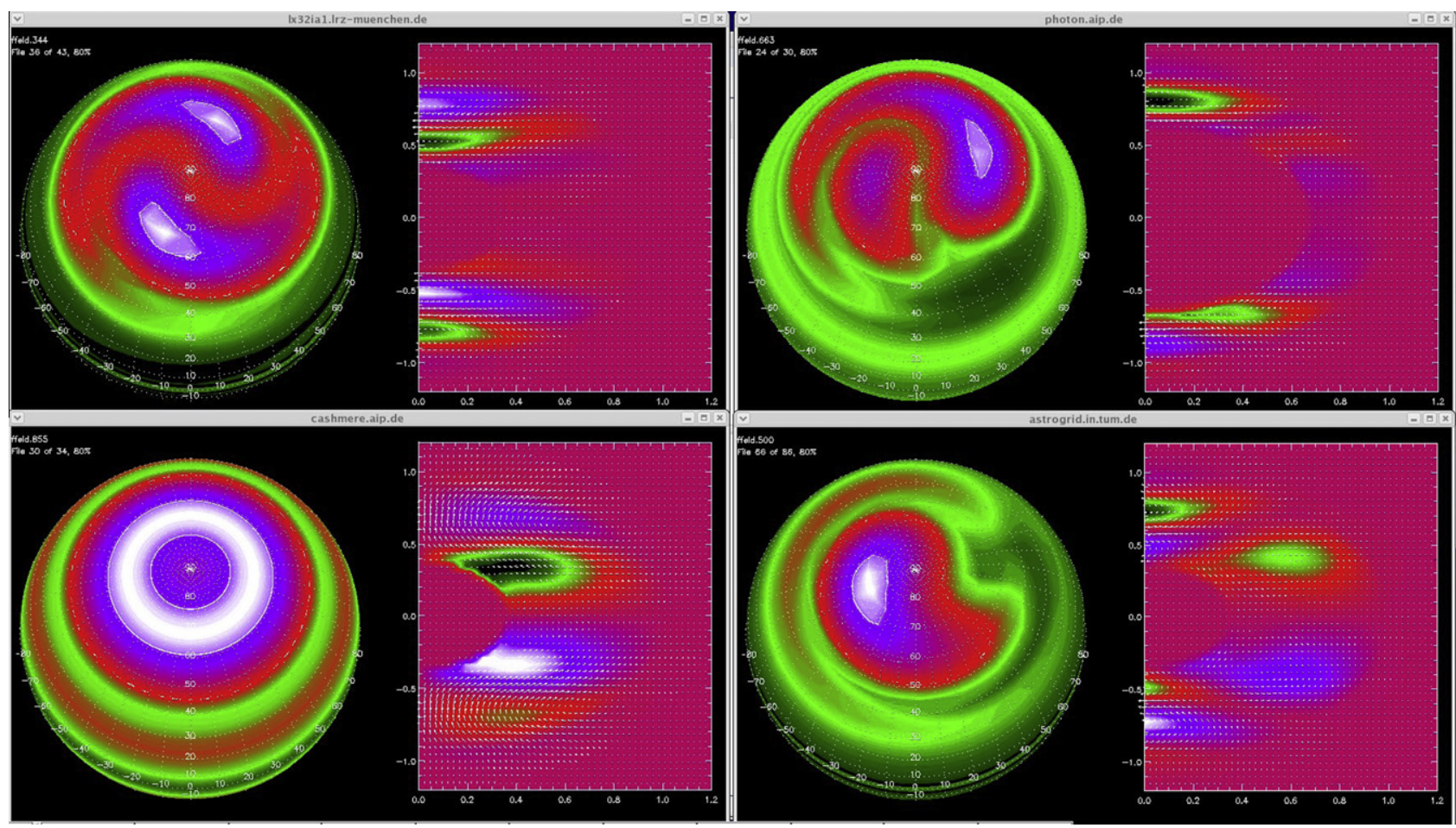

Fig. 1. Example output of a Dynamo run, showing real time results of four different grid resources. 
sources using single and parallel hardware, as well as parallel hardware with special purpose GRAPE cards. The ZAH offers the 32 node GRACE cluster (GRACE, WebLinks, 2010) as a resource of AstroGrid-D, with reconfigurable specialised hardware to a total peak speed of 4 Teraflop/s (Harfst et al., 2007; Spurzem et al., 2007, 2008). Another resource with GRAPE hardware integrated in the AstroGrid-D is a cluster at the Main Astronomical Observatory in Kiev, Ukraine (MAOKIEV, WebLinks, 2010), also an example of collaboration made possible on the basis of a grid Virtual Organisation.

Submission of an NBODY job starts with a shell script preparing an XML-based job description which is then staged and transported through the AstroGrid-D Globus middleware. Input data, output data and files go along with the job submission process. Future goals are to allow the submission of NBODY jobs through a portlet under the AstroGrid-D web portal and an integration of the AstroGrid-D file management system to allow handling of large datasets independent of the job staging process, see deployment instructions and tutorial (NBODY6++, WebLinks, 2010).

\subsubsection{GEO600}

The GEO600 use case is a task farming application. It uses the Einstein@Home application for analysing the data of the GEO600 Laser Interferometer near Hannover, in order to find signals of gravitational waves.

Einstein@Home is an ideal candidate for a grid application because of multi-platform support, well tested software base, simple resource requirements, built-in checkpoint and recovery methods, adjustable run time, and linear scaling with node number. Within the AstroGrid-D project we developed the software for grid deployment, job statistics and the details for constant production mode runs, such as restart after a regular job end and cleanup of recoverable errors.

The deployment is triggered by a script which is invoked in a Web Service Grid Resource Allocation and Management (WS-
GRAM) job to all grid machines on which the GEO600 jobs should run. As prerequisites on the target resource only Subversion (to retrieve the GEO600 source code) and a Perl interpreter are necessary. All other required software is installed during the deployment.

Depending on the number of currently pending and active tasks, the submission script will automatically determine when to submit new tasks to a grid resource. To establish a continuous submission scheme it is therefore sufficient to invoke the script periodically on the target.

The intermediate data are stored on the execution hosts since a central server approach would significantly slow down the job transfer rates. The submission of the GEO600 jobs can be controlled from a single workstation, from which the execution hosts are contacted directly. We plan to use the AstroGrid-D scheduler Gridway for the distributions of the GEO600 jobs in a future update. Furthermore it is foreseen to extend the GEO600 use case to grids based on other grid middlewares than Globus, such as gLite or Uniform Interface to Computing Resources (Unicore). This would allow a further distribution of the Einstein@Home jobs in the grids available.

The GEO600 use case has been running in production mode for more than a year, and it consumes around 1,00,000 CPU hours a day on D-Grid resources (see Fig. 2).

\subsection{Advanced applications}

Special purpose astrophysical applications and complex tool environments can also benefit from a grid infrastructure. We have chosen four relatively different use cases to represent this class of astrophysical applications and to show how we approach an implementation.

First, Clusterfinder is a use case involving both the deployment and performance of a typical compute-intense data analysis application and the extensive use of distributed data resources. Cactus shows how monitoring and steering methods for parallel

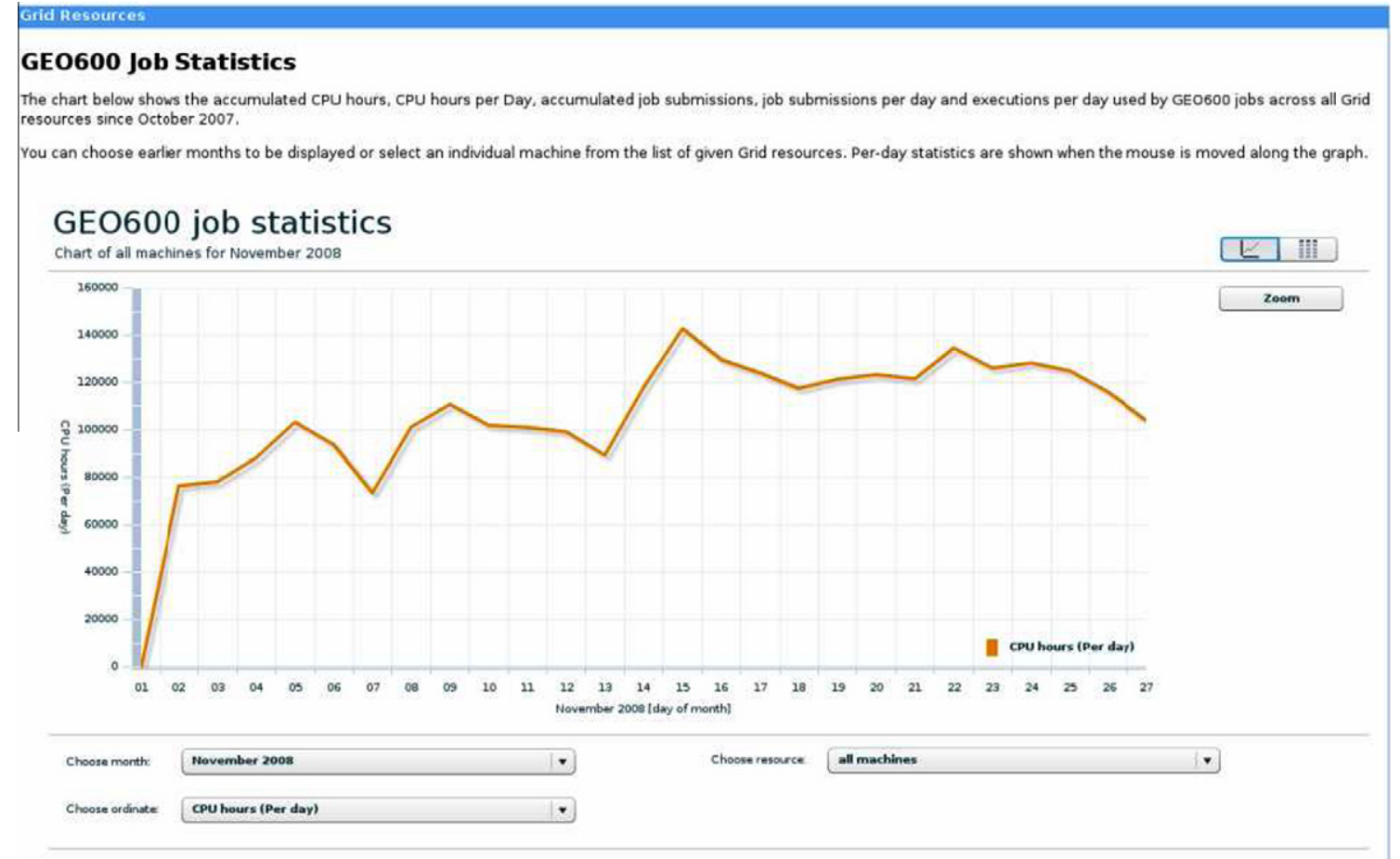

Fig. 2. GEO600 CPU Time November 2008, taken from (GEO600-statistics, WebLinks, 2010) with $x$-axis days of month, $y$-axis CPU hours consumed (sum over all used Grid resources). 
numerical simulations in the grid can be generalised, and how a web portal can provide user-friendly assessment of grid jobs and visualisation. Access to robotic telescopes as a grid resource represents a unique approach to a grid with heterogeneous elements. Finally, the Planck Process Coordinator Workflow Engine ProC has been grid-enabled to demonstrate the power of grid-computing when applied to the complex workflow of processing the data product of a satellite mission. It is a useful example for the handling of observation which may exceed the local capabilities or must be organised to suit the demands of a locally distributed working group.

\subsubsection{Clusterfinder}

Clusterfinder is an example for the deployment of a computeintense astrophysical application that uses distributed data, and its increase in performance. The scientific purpose of Clusterfinder is to reliably identify clusters of galaxies. It correlates the signature of X-ray images with that in catalogues of optical observations in order to study the large scale structure of the universe. Scanning at optical wavelengths to look for areas with an unusually large number of galaxies is not an unambiguous method to identify large clusters, as the galaxies may be spread out along the line of sight. Also the observation of the X-ray emission of the hot gas between galaxies will result in some false identifications as there are many other X-ray sources. In order to combine both sources of information, the theory of point processes is applied to calculate the statistical likelihood of a cluster at any point in space, and peaks in the combined likelihood are extracted into a catalogue of galaxy clusters (Clusterfinder, WebLinks, 2010).

Data retrieval and the calculations can easily be parallelised as the algorithm for any point in the sky depends only on data from nearby points, making Clusterfinder well-suited for grid implementation. Input of the Clusterfinder program consists of a cosmology and galaxy cluster model, together with the grid of sky coordinates and redshifts on which the likelihood is to be calculated. Scanning the available data consumes about 20,000 CPUhours per model. This entails over two years on a single processor or only several days when the resources of AstroGrid-D and D-Grid are used. An exploratory calculation on a smaller area can be executed on the grid in one night.

To implement Clusterfinder for a grid environment, two software tools were developed: A "grid-module" handles the installation and compilation on the resource, and an "environment" suite ensures that the necessary files and connections are available on any resource.

The logistics of performing Clusterfinder calculations on the grid involves splitting the calculation into jobs that can run in parallel, identifying grid hosts with the capacity to accept a job at the given time, reassembling the individual results into a coherent whole, and documenting the internal and external conditions under which the calculation was carried out. A single calculation is then submitted as a globus job and calculates a likelihood map with a given set of parameters. The results are collected using either the post-staging capabilities of Globus or by direct grid transfer using the globus-url-copy command. In the case of Clusterfinder, special consideration has been given to the input data. The SDSS and ROSAT all sky survey (RASS, WebLinks, 2010) catalogues are too large to copy the complete data set to a grid node. Therefore, the makefile controlling the Clusterfinder workflow is set up to request just the data needed from these catalogues.

A demonstration version of Clusterfinder is available as a portal application. The user can input coordinates and retrieve the corresponding likelihood map. It is planned to extend this portal to provide a production version of Clusterfinder as a grid service, including control over all the input parameters and even the files for the cosmological model.

\subsubsection{Cactus}

The Cactus Computational ToolKit (CCTK) (Cactus, WebLinks, 2010 ) is an open source, general purpose software framework designed to solve large-scale systems of partial differential equations on supercomputers using finite differencing techniques. In the Astrophysics science community Cactus is used to numerically simulate extremely massive bodies, such as neutron stars and black holes, and analyse the gravitational wave signal patterns emitted by these objects as predicted by Einstein's theory of General Relativity.

In AstroGrid-D we have developed application-specific techniques for Cactus which enable scientists to manage their simulations more efficiently and in a more collaborative context. Many of these methods make use of standard grid technology internally ( Deliv. 6.6, WebLinks, 2010).

As an example for online application monitoring and steering, users can connect to a running Cactus simulation just like any standard secure Hypertext Transfer Protocol (HTTP) web service, with a browser of their favorite choice. User authentication and authorisation is based on X.509 grid certificates (see Section 3.1.1). When logged in, users can query an up-to-date status of the simulation (e.g. the physical simulation time or stdout/stderr log output). Built-in online visualisation methods are available to analyse intermediate simulation data graphically via dynamic generation of 1D line or 2D surface plots, thus allowing users to evaluate the quality of the simulation while the application is still running. Once authorised, they can also steer the simulation by interactively changing parameters, triggering a checkpoint to be written, or by terminating the job gracefully.

Each Cactus simulation submitted to some supercomputer or grid resource can also announce itself at startup to the AstroGrid-D information service, by sending an RDF document with metadata uniquely describing the simulation. The information service is then able to keep a history of all simulations submitted by Cactus users. To access and search that simulation database we provide a Cactus portlet, based on GridSphere (see Section 3.1.4) as a standardised web interface. After logging into the portal, users can query the list of Cactus runs and filter it by owner, execution host, specific parameter settings etc. Queries are implemented as Cactus-specific GridSphere portlets (Deliv. 7.5, WebLinks, 2010), allowing the user to easily navigate through the list of simulations and browse individual query results. Also available in the portal are the results of nightly Cactus integration tests, which are performed automatically on various machines in the grid, in order to verify the correctness of the latest development version of the code.

\subsubsection{Robotic telescopes}

In recent years a growing number of ground-based robotic telescopes have been comissioned in astronomy, due to their increased technical reliability. Robotic astronomy allows observations from sites which may be astronomically favourable, but are otherwise remote or even hostile for human operators, e.g. Antarctica.

With more robotic telescopes becoming operational, there has been increasing interest in interconnecting them. Such a telescope network can accomplish new types of obervations. Examples are an uninterrupted observational campaign over many hours independent of day time and weather as required in astro-seismology, and rapid multi-wavelength observations in case of transient events.

AstroGrid-D contributes to this development with the (OpenTel, WebLinks, 2010) software package. OpenTel achieves the integration of robotic telescopes into the AstroGrid-D infrastructure and implements a telescope network based on grid middleware. Each telescope thus acts as an individual grid resource with its own grid certificate. One immediate advantage provided by grid technology 
is the direct connection to compute and storage resources for data analysis and archiving. Additionally, grid user and virtual organisation management provides a good solution for the central management of access rights.

The metadata management relies on Stellaris (cf. Section 3.2.1) and the (Usage Record format WebLinks, 2010) of the Global Grid Forum transformed into RDF. The metadata is retrieved from Stellaris using Simple Protocol and RDF Query Language (SPARQL) queries. The monitoring of observations is similar to the observation of jobs described below in Section 3.1.3. The Robotic Telescope Markup Language (RTML) (Hessman, 2006) of the Heterogeneous Telescope Network (HTN) (Allan et al., 2006) serves as the protocol for observation requests.

The OpenTel Tools package provides programs for the tasks of observation (job) submission, cancellation, and status queries. The programs are based on commands of the Globus Toolkit and are executed from the command line. Further details are described in (Deliv 5.3, WebLinks, 2010) and in the package documentation.

Several user interfaces have been developed to simplify operation management: the OpenTel Tools, the Telescope Map, the Telescope Timeline, a broker, and a scheduler. The Telescope Map is an interactive user interface shown in Fig. 3. It is an extension of the AstroGrid-D Resource Map (Section 3.1.3) for displaying geographic locations of telescopes and their properties such as available filters. Also displayed are day and night regions as well as weather information.

The Telescope Timeline is another interactive user interface useful for monitoring (Deliv. 2.7, WebLinks, 2010). It is an extension of the AstroGrid-D Timeline (3.1.3) and displays information about executed observations with an appearance similar to Fig. 7.

The broker achieves an automatic selection of telescopes based on the requirements of an observation (Deliv. 5.5, WebLinks, 2010). Filters and geographic coordinates but also the dynamic data such as the current weather conditions are examples for selection criteria.

The network scheduler generates observation schedules of the desired duration (Deliv. 5.8, WebLinks, 2010). Whenever necessary, an observation is handed over to be continued by another telescope of the network. An example for a $24 \mathrm{~h}$ observation of the star Gliese 586A (Gl586A) in the small network of Fig. 3 is shown in Fig. 4.

The OpenTel software has been tested with the AIP's robotic telescope STELLA-I (Strassmeier et al., 2004) and simulated networks. It is available at (OpenTel WebLinks, 2010).

\subsubsection{The ProC workflow engine for scientific grid-computing}

The Process Coordinator (ProC) is a scientific workflow engine. It was originally developed as an integral component of the software infrastructure for the Planck Surveyor satellite mission of the European Space Agency (Bennett et al., 2000).

Currently, two sets of scientific programs are being executed using the ProC, each forming a problem-domain specific toolbox. One is the simulation and data analysis package required for the Planck mission and cosmic microwave background (CMB) research (Reinecke et al., 2006). The other is a post-processing package for GADGET-simulations of cosmic structure formation (Springel, 2005), shown in Fig. 5. Both cosmological research areas are expected to benefit strongly from the parallel computing resources now being accessible for parameter space sampling problems via the grid-enabled ProC.

The ProC software package consists of three components: a graphical workflow editor, a graphical user interface for workflow execution, and a workflow engine, equipped with an application programing interface (API) and a versatile command line interface for expert users. The ProC is implemented platform independently in Java and uses the extensible markup language (XML).

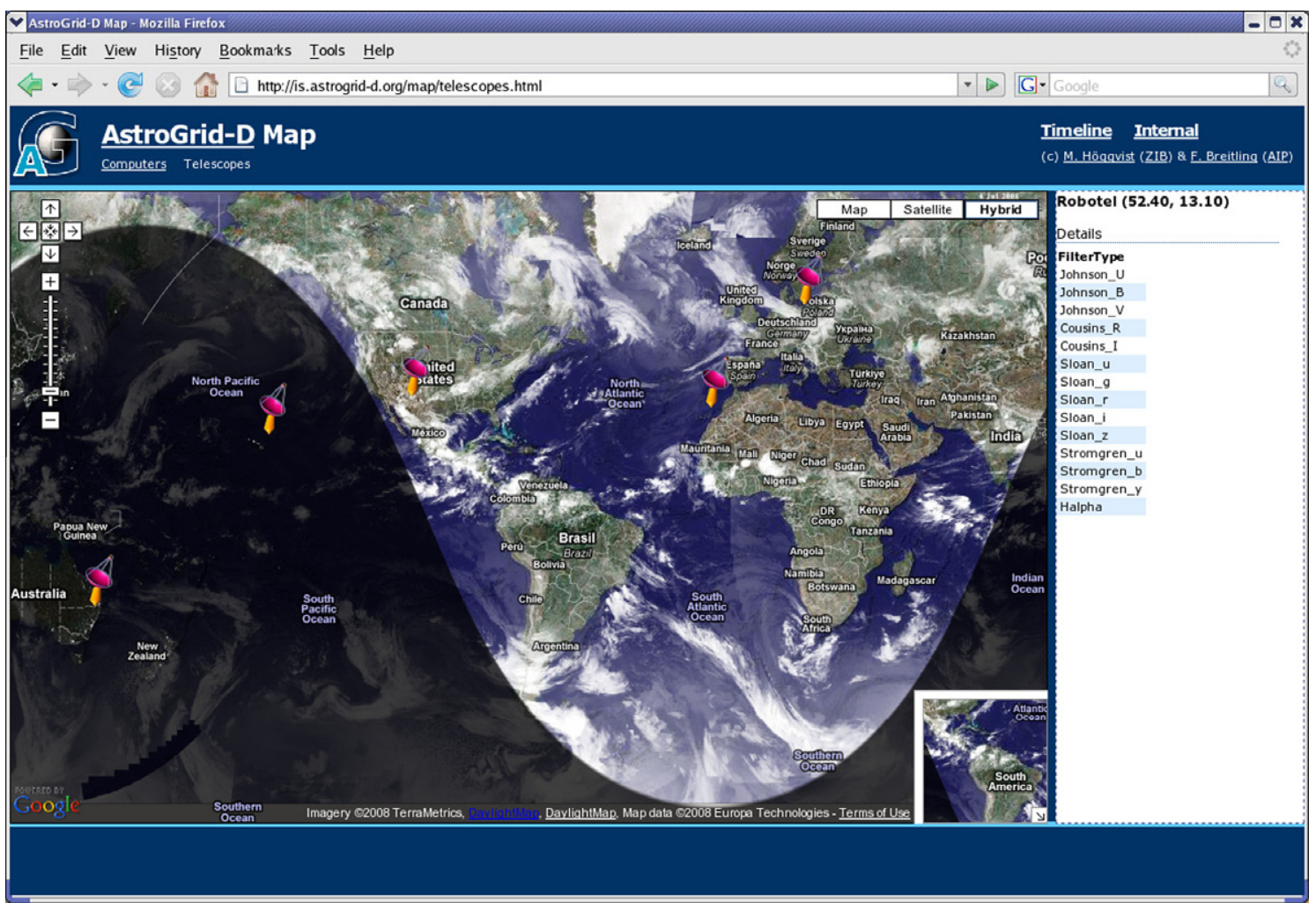

Fig. 3. The telescope map is an interactive user interface for the selection of telescopes. Daytime, weather conditions as well as the geographic location and properties of the telescopes are displayed. 


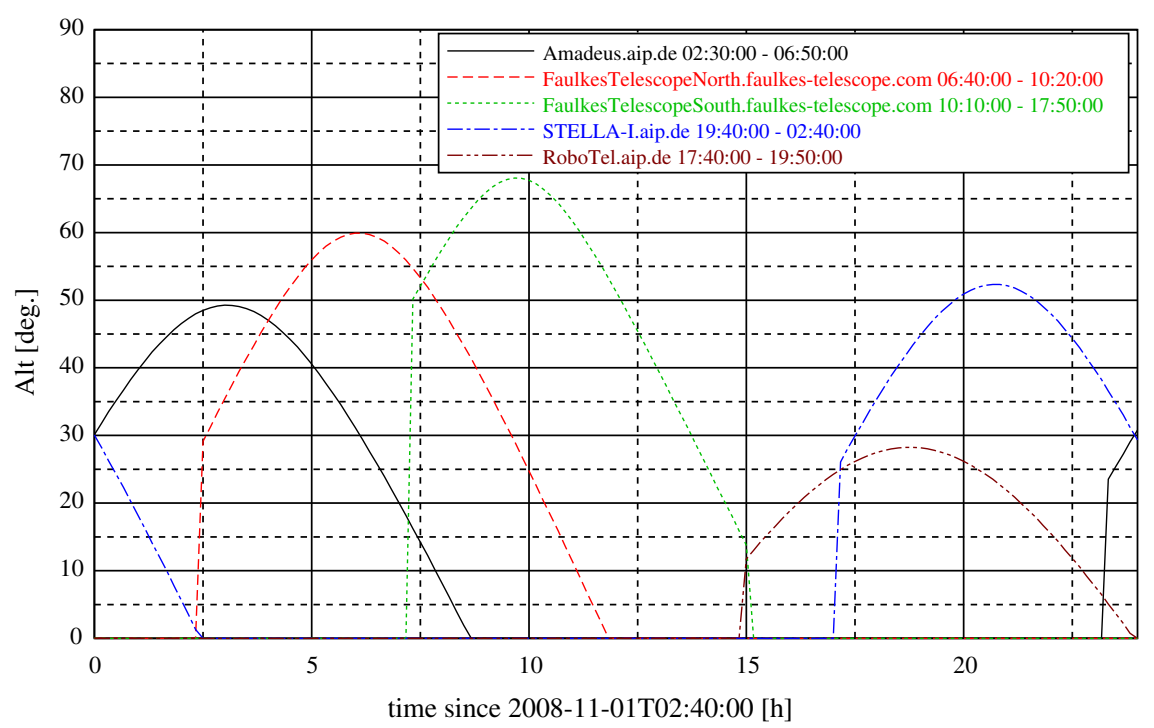

Observation: Gl586A (J2000 15.46 -9.34)

Fig. 4. Altitudes versus observation time for the network schedule of a simulated $24 \mathrm{~h}$ observation of Gl586A. The plot is produced by the OpenTel scheduler. The intersections of the altitude curves provide the time intervals for the observations by the different telescopes. Schedules are optimised for object altitude.

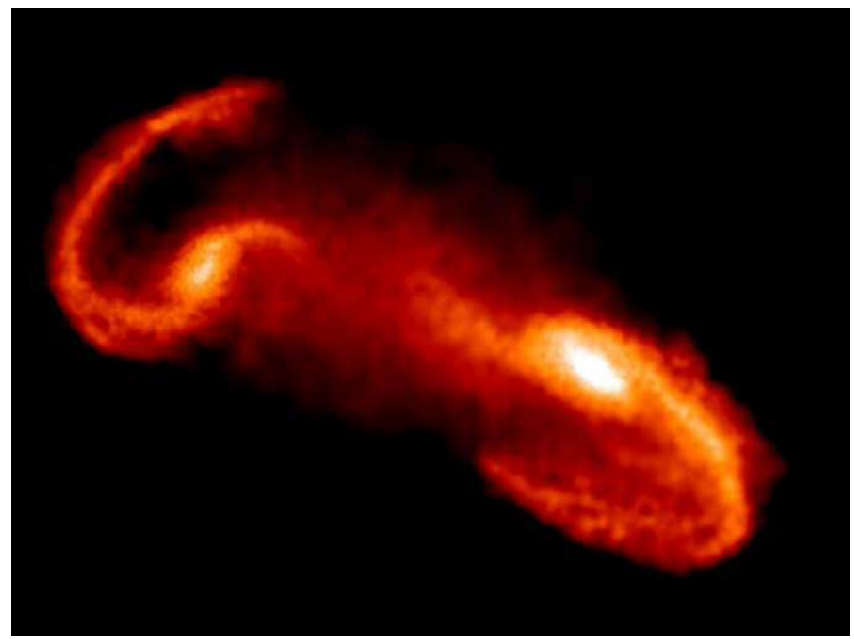

Fig. 5. ProC supported simulation: Galaxy collision calculated with the GADGETsimulation package steered by the ProC sampling control element.

One of the advantages of using the ProC, compared to simple scripting, is its ability to automatically recognize opportunities for reusage of previously generated computational results and for parallel execution of computational units. This latter capability can exploit multiple cores on a single processor, multiple processors cooperating in a local cluster, or the hundreds of compute elements offered by a dispersed grid.

With the help of the ProC Pipeline Editor the user is able to compose and modify scientific workflows consisting of programs, data flows, and control elements of the ProC library. Strong data-typing assures that only valid connections between modules can be made. The ProC's feature set includes typical control elements (e.g. loops), a fork/join mechanism, and specialised "sampling" elements for the investigation of high-dimensional parameter spaces via various algorithms. These elements permit user-controlled parallel execution of the same program segment on different data.

Within AstroGrid-D the ProC was grid-enabled with the help of the Grid Application Toolkit (GAT, cf. Section 3.1.4.1). In sample runs we used 200 compute elements simultaneously on a remote grid node. The need to deploy non-portable scientific code to a large number of grid nodes entailed the development of a comprehensive package of environment modules.

Upon request the ProC package is available free of charge for scientific computing purposes.

\section{The AstroGrid-D Services}

In this section we describe the architecture of our grid implementation and explain the role of several of its components and services.

We decided to base the astrophysical community grid on a recent version of Globus Toolkit (GT4) as a most widespread and advanced middleware solution. However, grid middleware capabilities are only generic functions and need enhancements to be of actual use. In more general terms the middleware serves as an abstraction layer or translation interface. It connects the resource (the individual hardware and its operating system) with the grid resource API (application programming interface) and with a set of uniform commands and applications, called the middleware $A P I$. The last interface is the one presented to the grid users and grid applications. An operational grid thus in some ways resembles a nonlocal operating system with enhanced capabilities, such as distributed storage or access to connected clusters and their batch systems.

In a second step we then modified or added architecture elements as necessary for Astronomical applications. The result is shown in Fig. 6. At the resource level we find compute elements $(\mathrm{CE})$, storage elements (SE), and instruments. While compute and storage elements are common to all grids and can be properly managed by the basic middleware, the inclusion of instruments (e.g. robotic telescopes) is one of the additions made by AstroGrid-D. Another addition is AstroGrid-D's central information service Stellaris (Section 3.2.1) which stores metadata of components, services and data (yellow ${ }^{1}$ block in Fig. 6).

We further extended the middleware capabilities for job and file management (green block in Fig. 6) by adding data stream

\footnotetext{
${ }^{1}$ For interpretation of colour in Fig. 6, the reader is referred to the web version of this article.
} 


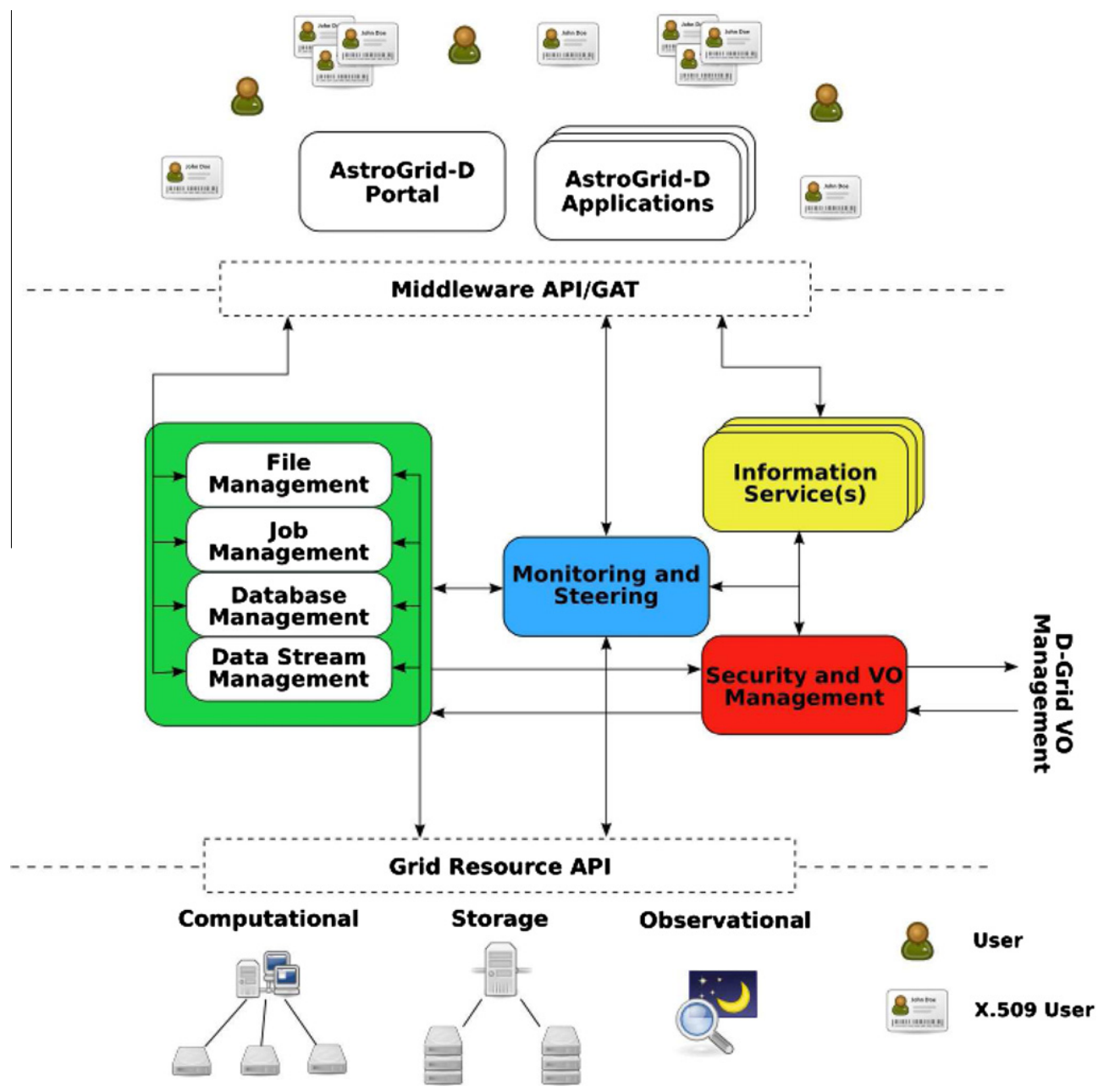

Fig. 6. Sketch of AstroGrid-D architecture, showing the layers and services that are involved in a grid application, and the pathes of interaction.

management (Section 3.2.3. Other components were enhanced: Monitoring and steering were attached to the Stellaris information service (blue block in Fig. 6). With our Virtual Organisation management we achieved user and group management based on the GT4 security layer (red block in Fig. 6), to implement a grid that can easily be used by collaborations to share access rights and data.

Fig. 6 only illustrates the architecture components. Not shown is the underlying, interconnecting network and the security layer.

\subsection{Working with AstroGrid-D resources}

Each system with built-in security requires the user and even services (hosts, databases etc.) to authenticate themselves. The grid uses X509-certificates, i.e. public/private key encryption, for this purpose. At least one Grid Certification Authority per country provides such certificates for users, resources and services. With this certificate it is possible to log onto other grid resources from any grid-enabled workstation.

The following subsections describe some details of grid and resource work. The subsection about VOrg Management shows how the collaborative concept of virtual organisations and the security layer are tied together. Then, a brief overview of the procedures for integrating a resource into the grid is provided. The last paragraphs introduce different interfaces provided by AstroGrid-D.

\subsubsection{VO Management}

Virtual Organisations (VOrgs, often somewhat confusingly called VO's) are a central element of any grid. In some aspects they are the grid representation of the more familiar "group" concept of an operating system. A VOrg is formed by any number of users with a common intention to share resources, data and access rights in a grid.

In AstroGrid-D any user is authenticated by an individual X.509 certificate. However, the certificate itself does not allow access to resources of AstroGrid-D or D-Grid, since that right is restricted to members of our main VOrg "AstroGrid-D". Thus each user must also register for membership to this VOrg.

To improve the registration process and administer the members, AstroGrid-D uses a service written by Fermilab, the Virtual Organisation Membership Registration Service, (VOMRS, WebLinks, 2010). The registration service itself is only accessible with the user's certificate installed in the web browser. During the registration process some of the user's work details are collected, such as name and institution. The user also has to choose which of the available VOrgs he wants to belong to. Upon verification by the user's institute, the VOrg Administrator will grant the membership status.

Additionally to the main VOrg, in AstroGrid-D currently four smaller VOrgs exist. These Sub-Organisations are used by specific institutes for internal grids, for our robotic telescope resources and our collaboration with GAVO.

To connect the VOrg member database of AstroGrid-D with each resource, we developed a separate service. At each resource this service regularly queries the central VOrg database for changes, and the resulting user list is applied to the resource's local access management. When an accepted VOrg member then logs 


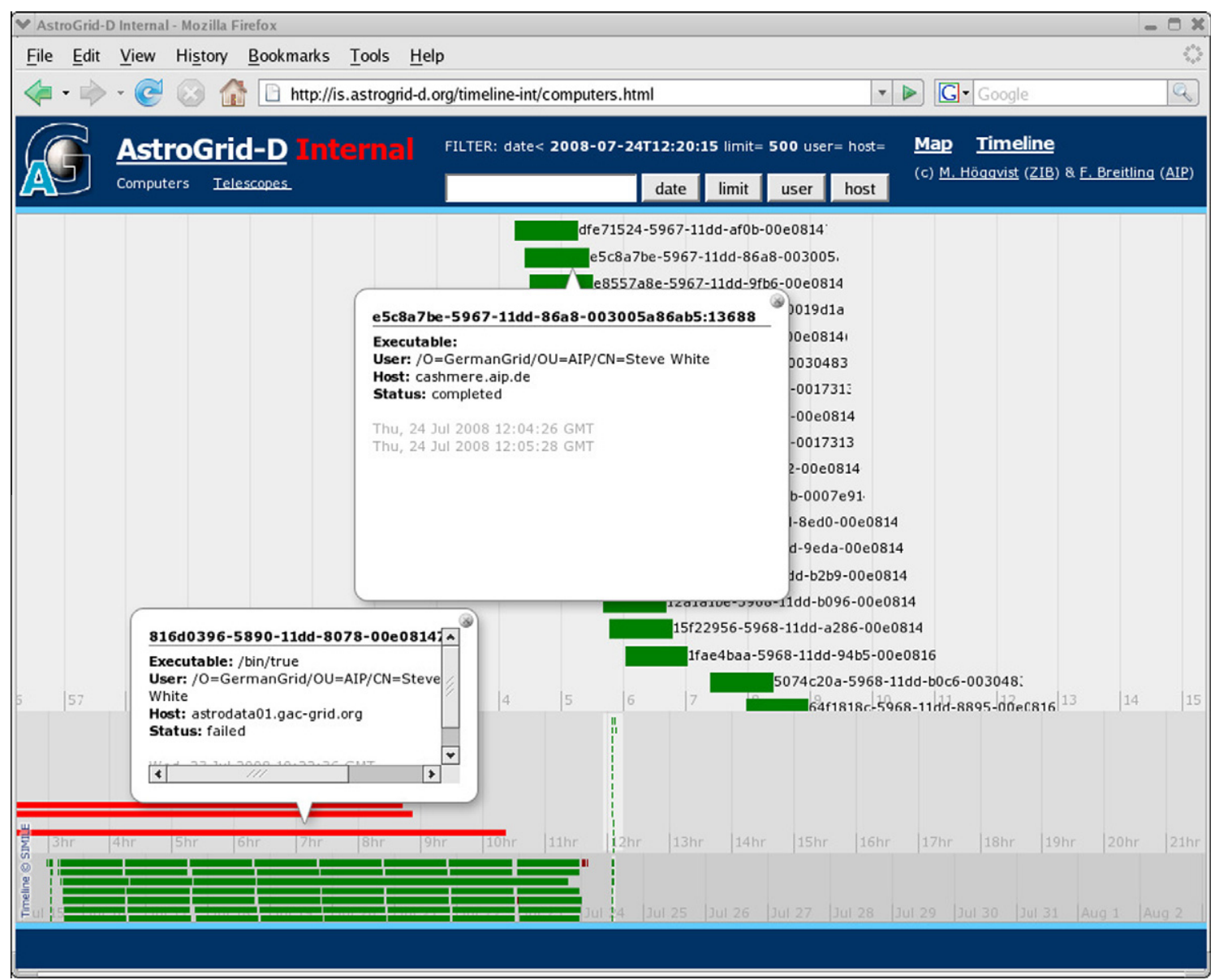

Fig. 7. The Timeline is an interactive user interface for displaying status, progress and general information of grid jobs. The top area displays hours, each line displaying the duration of a job and an identifyer. A mouseclick opens up an information window (inset), displaying indepth information about the job. In the below areas the scope of display is days and months.

onto the resource and is properly authenticated by the Globus Toolkit, he is mapped to an individual, local UNIX user account.

Our extension to the VOMRS offers a number of options for local resource administrators, e.g. to import only specific VOrgs or white- or blacklist single users. The system also supports OGSADAI (see Section 3.2.3) and Unicore user formats and cluster options. Individuals who change their "distinguished name" string, e.g. due to a change of institution, can be mapped back to their former grid account. Even if there is in general no guarantee for user data to persist in the grid, it is often useful to re-gain an existing environment of local settings and libraries.

AstroGrid-D established the VORMS based solution in 2006. Since then it was stable in operation, managing the about 100 users of AstroGrid-D. The successful concept was then also adopted by the German D-Grid where it became the standard form of user management.

\subsubsection{Resource integration}

AstroGrid-D is currently comprised of about 20 grid resources provided by its member institutions: computer clusters, workstations, data storage servers, as well as a telescope server.

German astronomers apply for inclusion of a computer resource into AstroGrid-D on an individual basis; all German academic institutions are eligible by default. Resources of an Ukrainian institution have also been included for collaboration.

Ideally, to bring a resource onto the grid takes about fifteen hours for an experienced administrator. In practice more time may be required, due to complications in networking, retrieving certificates, and operating system peculiarities. Why would an institute invest that work and put their valuable computer resources on the grid? First, there is anyway considerable overhead for sharing of resources between institutions: accounts have to be set up, ports opened for special communications, etc. These problems are solved by bringing resources onto the grid and using the tools and standard solutions it provides. Second, on the grid, a resource has a much wider group of users and can be used to full potential.

All steps required to bring hosts online as AstroGrid-D resources are described at (AGD-Globus, WebLinks, 2010).

\subsubsection{Monitoring}

In a distributed, diverse grid environment, the monitoring of its parts and processes is of central importance for users and administrators. Monitoring can in principle be divided in two categories: resource and job monitoring.

Resource monitoring for compute and storage resources is realised in AstroGrid-D through the Monitoring and Discovery System (MDS) of the Globus Toolkit. MDS is a suite of web services to monitor and discover resources and services on Grids. The gathered information is displayed on the AstroGrid-D resources overview web page (MDS, WebLinks, 2010). An independent monitoring mechanism has been developed for telescope resources, which handles telescope-specific information such as weather.

As a complementary interface to the resource list view, AstroGrid-D has developed a resource map as an advanced user interface for displaying collected resource information topographically. The 
Telescope Map in Fig. 3, discussed in Section 2.2.3, is a specialisation for telescopes. Both are based on the Google maps API. When a resource is selected, additional information about its load and usage is displayed. The information is obtained via SPARQL queries from (Stellaris, WebLinks, 2010), after it has been extracted from MDS, converted into RDF and uploaded to Stellaris. The Resource Map can be accessed at AGResourceMap, (WebLinks, 2010). The software can be obtained from the AstroGrid-D web page.

Job monitoring is based on globus' audit logging. Audit logging writes job status information into a database. This information is translated into RDF/XML and transferred to Stellaris.

The AstroGrid-D timeline was developed as a plain user interface to job information. It is based on the (simile timeline WebLinks, 2010). Jobs are represented by horizontal lines of length proportional to the job duration. A colour code represents the status. For each job, additional information such as user ID and name of executable can be displayed. The search for information can be limited with keywords and in the public area the details are strongly reduced for privacy reasons.

Further details about monitoring can be found in (Deliv. 5.9, WebLinks, 2010).

\subsubsection{User and developer interfaces}

In AstroGrid-D there are four different ways available for actual grid use. The middleware itself provides a commandline interface as well as an API for software. The Grid Application Tool (GAT) provides an alternative API which hides the underlying grid middleware and makes its use transparent. And finally, GridSphere enables developers to quickly develop portlets for grid applications. Both GAT and GridSphere do not require the installation of a grid middleware on the submission host, and it is also possible to use them on Windows machines.

3.1.4.1. The globus commandline and API. The AstroGrid-D resources are grid-enabled by Globus middleware. They can thus be accessed via the command line interface of Globus. This interface allows data transfers and submission of jobs to the grid and provides many more operations. For applications, Globus offers a rich API for each component of the middleware.

The Grid Application Toolkit (GAT, WebLinks, 2010) is an API which offers grid access irrespective of the middleware which con- nects the resource to the grid. The GAT Engine and preliminary adaptors have been developed as part of the EU funded (Gridlab, WebLinks, 2010). Within the AstroGrid-D project the Java implementation of JavaGAT is used. AstroGrid-D added adaptors for SGE, PBS, WS-GRAM and gLite (see Fig. 8), and recently also a UNICORE adaptor (UNICORE 6) was contributed by the DGI-2 project. JavaGAT currently features adaptors to all the grid middlewares, which are used in D-Grid. JavaGAT uses the security layers of the middleware.

The availability of "local adaptors" enables the programmer to develop the application logic without a connection to the grid. The developed application has then access to all grid middlewares for which JavaGAT adaptors are available.

3.1.4.2. GridSphere. Like GAT, (GridSphere, WebLinks, 2010) was developed as part of Gridlab in 2002. The main goal of the portal related work focused on building a reliable, structured web interface to support the European and global grid community. A portal application can store the specifics of a grid job and run it from any standard Web browser. GridSphere is JSR 168 compliant and thus portlets running in GridSphere can run as well in other portal frameworks.

GridSphere comes with a variety of core portlets providing all the basic functionality, such as profile personalisation, layout customisation and administrative use.

The GridSphere AstroGrid-D portal offers a portlet for Clusterfinder, and a Cactus Portlet is available at AEI.

\subsection{Components of the AstroGrid-D architecture}

The middleware of the AstroGrid-D builds on existing grid tools to integrate diverse types of resources. To accommodate the specific requirements of the AstroGrid-D community, existing components were extended or substituted by newly developed ones. However, to let other communities benefit from these developments, we aim at generic solutions wherever possible.

The following subsections describe (1) the information service Stellaris, for central storage of all metadata and status information, (2) enhanced data storage capabilities of the grid, (3) grid access to data sources, efficient data transport and data streams, and (4) options for job submission.

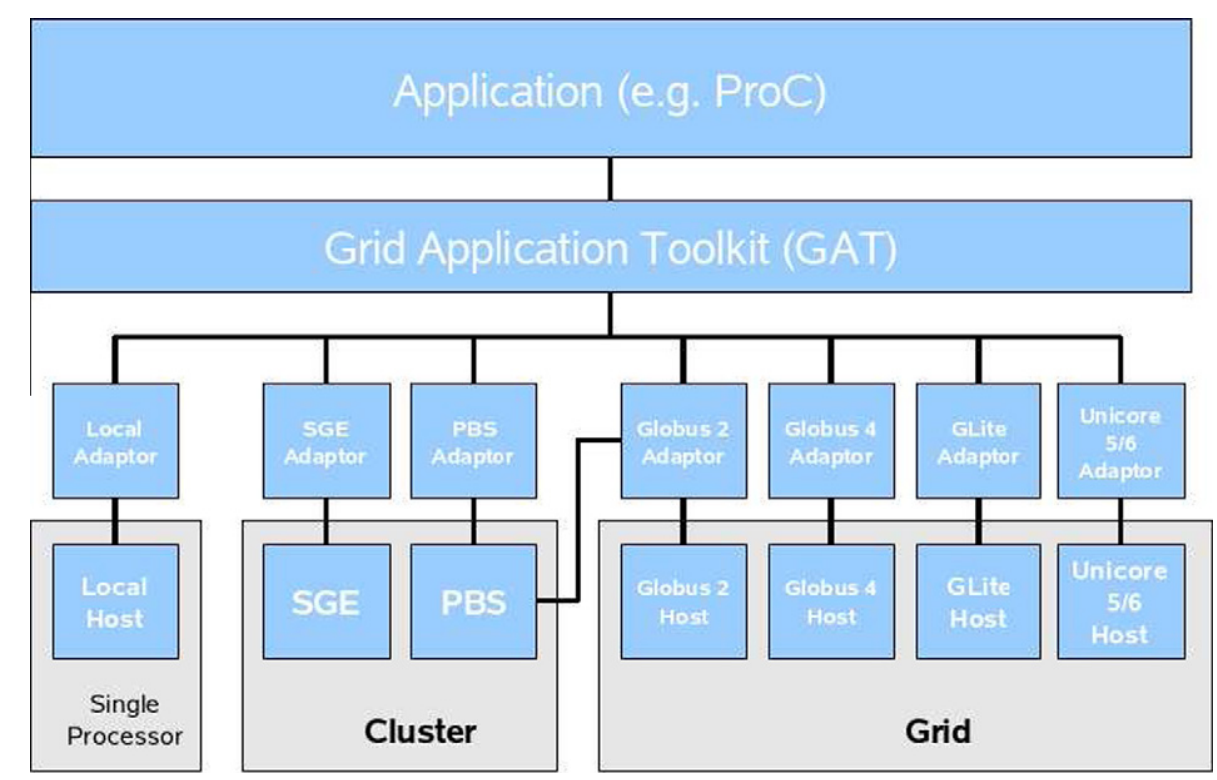

Fig. 8. JavaGAT Architecture. 


\subsubsection{Information service}

The goal of the AstroGrid-D information service, Stellaris (Högqvist et al., 2007), is to provide a uniform framework for storage and querying of grid related information and metadata. Typical usage scenarios result in questions such as: Was dataset $X$ already analysed with program $Y$ and parameter set $Z$ ? Where is the output data from August 12th last year? Why did my last grid job fail? Who created the data producing the graph from the latest number of Science and where can I find it?

Within AstroGrid-D, we distinguish between four different types of metadata: (1) resource metadata describes properties of the shared resources (e.g. for a telescope the aperture, filters, ccd, capabilities), (2) activity state reflects the current and logged state of activities in the grid such as the location and characteristics of jobs and file transfers (e.g. user, name of telescope, its location, start and end of observation, priority), (3) application metadata describes the program and its input parameters (e.g. RA/Dec of the target, requested filters, etc.), and (4) scientific metadata, which includes information about the provenance of datasets which are used (science project, type of data (image, table), provenance, references, etc.). In order to respond to the previously stated example questions we will often need to query metadata of more than one of the information types. Therefore, the integration of metadata from many different sources is a strong requirement on the information service. We solve this problem by using the common metadata model ( $R D F$, WebLinks, 2010) for all the information types.

The information system architecture in AstroGrid-D (see Fig. 9) consists of three main components; Stellaris, the information service, data producers (applications, grid resources, and services) and data consumers (applications, services and users). The Stellaris service itself is designed around two World Wide Web Consortium (W3C) standards: RDF for metadata representation and (SPARQL, WebLinks, 2010) which is used for querying the information service. Thereby, we can benefit from existing tools for e.g. data integration and visualisation developed by the web-community at large. The (Stellaris software, WebLinks, 2010) was developed within the AstroGrid-D project and is made available under the Apache Open Source license.

\subsubsection{File management}

The AstroGrid-D Data Management (ADM) has been developed as a tool for distributed file management. It offers access to the user's files through the concept of a virtual file system via the command line, a web interface, or a programming interface. Globus contains a software tool denoted as Globus Replica Location Service (RLS), which allows to manage file replicas across the grid re- sources. We found the latter to be somewhat difficult to use with job submission through the GridWay service to an execution host, whose selection is not directly controlled by the user. Our ADM system delivers proper software tools to identify files and tag them with metadata independent of the original job execution environment. This is especially useful if the user needs to deploy data files required for job start and to access files after a job execution for post-processing.

ADM uses a relational database to store a unique file descriptor, i.e. a logical file identifier for each file, plus meta data for each file or directory, e.g. the owner and a timestamp to log when the entry has been registered with the filesystem. While file ownership and creation timestamp are mandatory, and ADM transparently cares for their maintenance, meta data and individual files can be endowed with custom (user-defined) properties. ADM provides the command line client adm, including a C-library, which offers an easy-to-use access to the stored files. Furthermore, ADM ships with a web interface which permits to browse the virtual filesystem graphically.

\subsubsection{Data base access and data stream management}

Access to databases storing observational and simulation data has become an important part of daily astronomical work. Depending on the various application requirements and data characteristics, databases store the actual raw measurements, results and/or the according metadata. AstroGrid-D considers it a major task to develop database technology further for building scalable data management infrastructures. We are motivated by a growing number of users and especially the expected data rates of forthcoming projects, such as the Panoramic Survey Telescope and Rapid Response System (Pan-STARRS) or LOFAR.

Due to the distributed nature of data sets and research groups, using a grid-based approach is a natural choice for the astrophysics community. The Open Grid Services Architecture-Data Access and Integration (OGSA-DAI, WebLinks, 2010) services enable the integration of databases in grid environments and they are part of the Globus grid middleware. Therefore we chose OGSA-DAI to provide database data on resources within the AstroGrid-D and D-Grid infrastructure. Fig. 10 gives an overview of the AstroGrid-D database management.

In order to reduce the network traffic induced by distributed queries on various data sources and to achieve load-balancing within the community grid, various load-balancing techniques have been tested and evaluated (Scholl et al., 2007a,b, 2009a,b).

Especially data-centric applications, such as the Clusterfinder use case (Section 2.2.1), benefit from the increased throughput

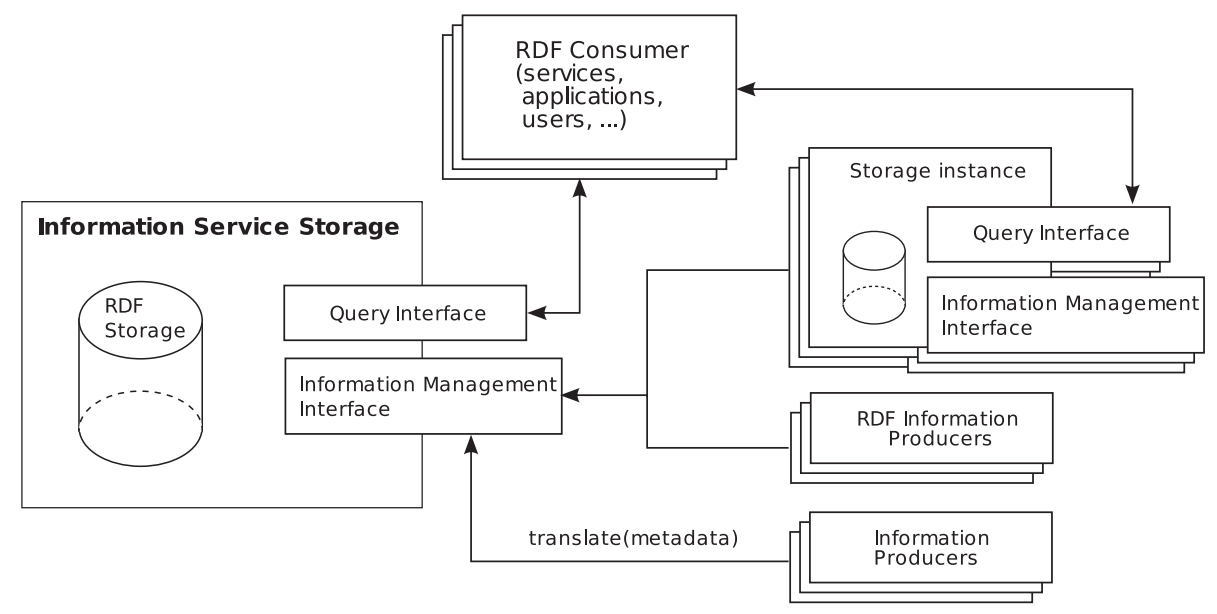

Fig. 9. The AstroGrid-D information service framework. 


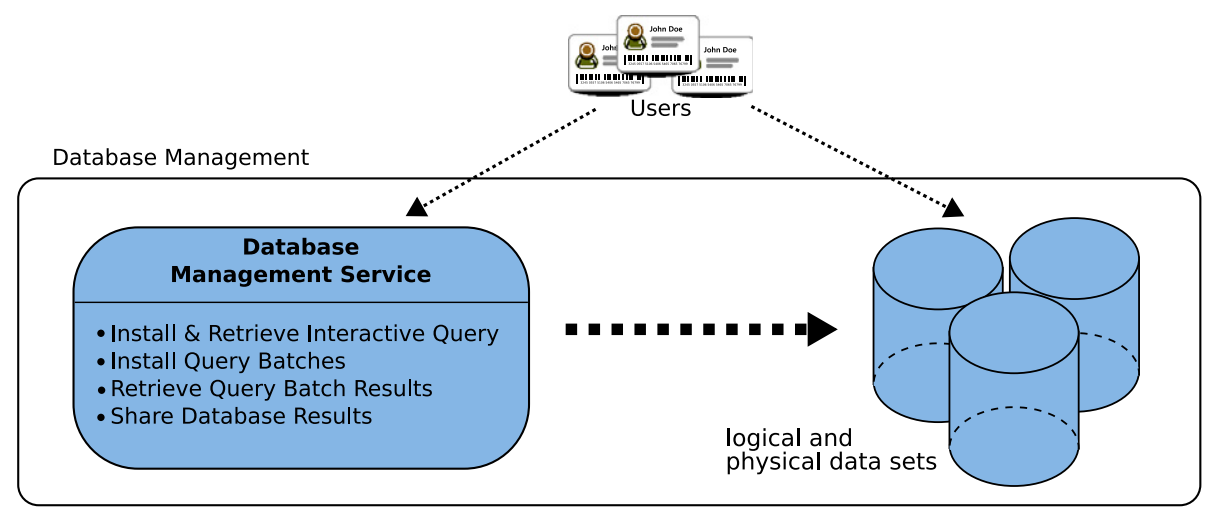

Fig. 10. The AstroGrid-D database management. Users can access the data sets both interactively and with batch jobs. The actual nodes on which the data sets reside is kept transparently.

introduced by load-balancing techniques for their database accesses (in the case of Clusterfinder to the SDSS and ROSAT databases). The database relations have a fixed schema, which is also available via the metadata of the database system used. Data access and manipulation is performed via the standardised query language SQL. In future we also plan to support the Virtual Observatory Query Language (VOQL, formerly ADQL, WebLinks, 2010)), a specialised query language for astronomical data based on SQL and an important effort by the International Virtual Observatory Alliance (IVOA).

Another prevalent processing model for e-Science data are data streams. Sensor sources (e.g. telescopes, satellites) continuously generate such data output. Due to the fundamental importance of these sensors within astrophysics, we investigate efficient data stream processing models within AstroGrid-D. An important initial processing step of data streams is data filtering. Existing middleware structures do not offer such a processing model (yet).

XML or XML-based protocols are the de facto communication standard for web services and as well many astronomical IVOA protocols. Therefore, AstroGrid-D uses XML-based processing of data streams that are published by data sources and scientific applications can subscribe to. In order to increase the reusability of data streams for multiple subscriptions, the query processing is performed by installing individual processing steps (operators) within the grid network.

Running a data stream management within astrophysics requires means to define and commonly share scientific operators based on already implemented functionality. A reusable operator is e.g. a chi-squared filter for configurable thresholds for quality assurement. Mobile operator repositories enable researchers to provide these operators via their own institution (e.g. personal web page) and to describe the operators with appropriate metadata in the information service (Section 3.2.1). This considerably facilitates collaborating researchers to discover and reuse such existing operators. Signing the operators with the author's grid certificate allows users to verify the trustworthiness of the operator's source.

Techniques such as early filtering and early aggregation lead to good results, especially in the context of multi-subscription optimisation (Kuntschke et al., 2005; Kuntschke and Kemper, 2006a,b). The AstroGrid-D data stream management (see Fig. 11) is available on all AstroGrid-D resources.

By developing data stream processing techniques for grid environments, we moreover support the conversion from persistent data sets to streams. A combined, integrated processing of persistent and streaming data, as required by applications such as SED classification, is possible and results in better performance (Kuntschke et al., 2006).

\subsubsection{Job management}

AstroGrid-D has implemented job management through the independently developed (GridWay WebLinks, 2010) Metascheduler on top of the standard globus middleware layer. As a metascheduler, GridWay enables large-scale, reliable and efficient sharing of computing resources managed by different Local Resource Management (LRM) systems, such as the Portable Batch System (PBS), the Sun Grid Engine (SGE), or the LSF, within a single organisation (enterprise grid) or scattered across several administrative

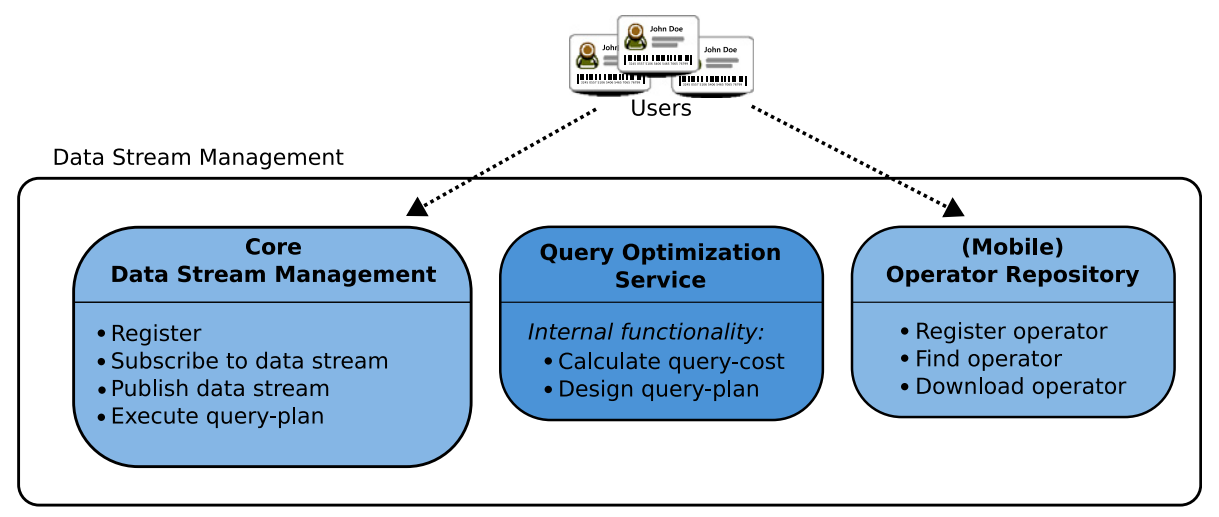

Fig. 11. The AstroGrid-D data stream management. Users can publish and subscribe to data streams and share their stream-enabled operators using operator repositories. Internally, the data stream services provide optimisation capabilities. An example of an operator would be a function performing a RA/DEC transformation into various coordinate systems. Another example operator would be a Java program listening for specific data from a data stream of an instrument source. 
domains. In the second case GridWay can interact also with other grid middleware than Globus, such as e.g. Unicore or gLite. GridWay is meanwhile fully integrated into the globus open source project, adheres to Globus philosophy and guidelines for collaborative development and so welcomes code and support contributions. GridWay has its own set of line mode commands, such as e.g. gwsubmit, gwstat or gwhosts to control the available resources and one's own jobs. GridWay can serve as a comfortable user interface to the entire grid, similar in style to a local resource management system (LRM, queue system). Note that resource informations have to be provided through the Globus MDS information service and middleware to the GridWay server. The LRM "Fork" means that single processor jobs are accepted to be started by a Unix process fork. Another LRM available is PBS (portable batch system) for parallel jobs. Fig. 12 illustrates three stages of a job run, for the example of an NBODY calculation (Section 2.1.2). The first step is the deployment which delivers an XML based job description as described in Section 2.1.2. Such XML jobs can be submitted through the standard Globus GRAM job submission interface and middleware to the Gridway host rather than directly to the LRM of an execution host.

Gridway then receives this job through Globus and the Gridway Job Manager acts as a broker and scheduler. It selects an available execution host through a matchmaking process and submits the job to it by Globus GRAM. At present we have implemented a simple round robin strategy for single fork jobs; the GridWay software in principle allows to implement more complex scheduling algorithms including user defined parameters. It is always possible to submit jobs targeted to a certain resource through GridWay, though this is not the desired mode of operation.

The third step is the execution and post-processing stage, during which it has to be ensured that the build process properly works on the target resource and that the user receives the simulation results for post-processing.

The two- step submission procedure with two Globus GRAM jobs connected by the GridWay server is denoted as GridGateWay. Note that it is also possible for the user to directly logon to the GridWay host and use it for job submission directly.

\section{Summary and outlook}

\subsection{Summary}

AstroGrid-D established a nation-wide pool of compute, data, and instrument resources accessible for astronomers. It also integrated special hardware compute resources like clusters of GRAPE6 boards into the grid. The use case NBODY6++ shows impressively it's exploit in a grid environment. Well documented procedures explaining how to bring a resource into the grid are available. Authentication and authorisation for the use of the grid resources is managed by the Virtual Organisation. Moreover, the resources of AstroGrid-D were integrated in D-Grid, which in turn provides access to the resources of the whole D-Grid for the AstroGrid-D members. Robotic telescopes were also integrated into the grid as a special hardware resource, so they can be accessed like any other compute node.

A variety of typical astronomical applications was brought to the grid. We investigated simple but compute-intensive task farming applications like Dynamo or GEO600 and showed that it is very easy to run them on the grid without the need of complex reprogramming. We also looked into more complex and data intensive tasks like e.g. the Clusterfinder and ported them to the grid. The Clusterfinder program, e.g. is now able to scan the entire available data for one model parameter set within several days, whereas it would need more than two years on a single processor.

We developed a set of high-level services: Programmers can now make use of an information service to handle meta data and to monitor jobs and resources. Also, they can abstract from interfacing a specific grid middleware and use GAT instead. Moreover, GridSphere enables a user friendly grid access with any web browser. The ProC workflow engine supports the composition of scientific workflows and their parallel grid execution. Resource brokering and job scheduling is augmented in AstroGrid-D by the GridWay Metascheduler. Thereby more complex scheduling algorithms can be implemented. The AstroGrid-D Data Management ADM handles file staging in combination with the job submission via GridWay. It thus provides an easy-to-use access to stored files

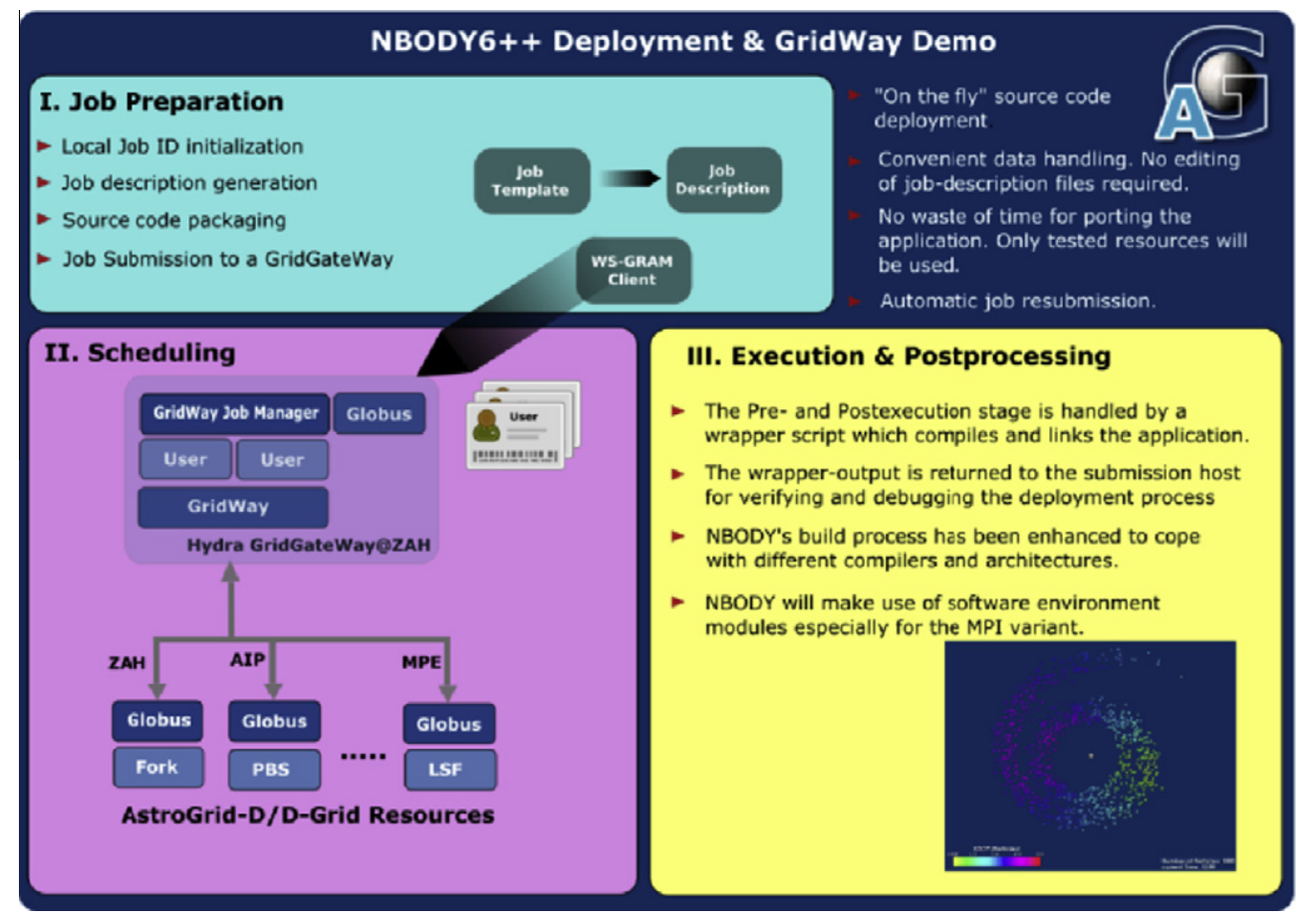

Fig. 12. Flowchart of steps to submit NBODY job via GridWay. 
and their replica in the grid. The integration of databases and data streams is also provided by AstroGrid-D. Special attention is paid to optimising techniques that guarantee good performance results as well for throughput as for response time. Many of the services summarised above are addressed in close collaboration with GAVO, whose focus is more on the side of the scientific user, whereas AstroGrid-D is solving the technical and infrastructural aspects.

Most of the German community grids, except the High Energy Physics community, employ the Globus Middleware. On EU level, gLite developed by EGEE is dominating all grid efforts, whereas internationally, the split is equal between EGEE/gLite and Globus. A lot of effort goes into interoperability of these different middlewares, but sometimes there still are barriers. AstroGrid-D is collaborating with both EGEE/EGI as well as the Open Science Grid (OSG).

\subsection{Outlook}

The important next step is to enlarge the community of grid users. For this purpose, the consulting and the support of new users has to be professionalised. We are able to offer considerable resources in compute power and storage to the scientific community.

There are some infrastructure elements that we would like to improve, e.g. our methods for resource brokering and job scheduling.

Proper and efficient handling of large amounts of data is a key feature that the grid offers. Upcoming projects such as LOFAR, PanStarrs or LSST will produce immense data volumes whose storage, administration, and processing can no longer be handled by local institutions. Moreover, this data is in many cases processed in distributed, international working groups. Grid technology is an appropriate answer to these new challenges. Due to the parallelisation potential and the security layers of the grid, administration and access can be achieved even in a complexity where central processing hits its limit. For this purpose we need a powerful data management component to enable handling files, data bases, and data streams in a coherent framework.

AstroGrid-D established a solid basis to cope with these future challenges arising from forthcoming scientific needs. We are looking forward to establish our solutions as a cornerstone of German e-Astronomy.

\section{Acknowledgments}

This work is supported by the German Federal Ministry of Education and Research within the D-Grid initiative under contracts 01AK804[A-G]. AIP acknowledges support by EFRE, Grant No. 9053 ARI-ZAH acknowledges support of the GRACE project by Volkswagen Foundation Grant No. I/80 041-043 (Project 'GRACE') and by the Ministry of Science, Research and the Arts of BadenWürttemberg (Az: 823.219-439/30 and /36). We acknowledge the special memorandum of understanding between Astrogrid-D and the astronomical segment of Ukrainian Academic GRID Network. We thank Ignacio Llorente, Ruben Montero, and Tino Vázquez of Universidad Complutense Madrid, Spain, for help and support in installation and operation of the GridWay service.

\section{References}

Aarseth, S.J., 1999. From NBODY1 to NBODY6: the growth of an industry. PASP 111 1333-1346.

Allan, A., Hessman, F., Bischoff, K., Burgdorf, M., Cavanagh, B., Christian, D., Clay, N. Dickens, R., Economou, F., Fadavi, M., Fraser, S., Granzer, T., Grosvenor, S. Jenness, T., Koratkar, A., Lehner, M., Mottram, C., Naylor, T., Saunders, E., Solomos, N., Steele, I., Tuparev, G., Vestrand, T., White, R., Yost, S., 2006. A protocol standard for heterogeneous telescope networks. Astronomische Nachrichten 327 (September), 744

Bennett, K., Pasian, F., Sygnet, J.-F., Banday, A.J., Bartelmann, M., Gispert, R., Hazell, A., O'Mullane, W., Vuerli, C., 2000. Sharing data, information, and software for the ESA Planck mission: the IDIS prototype. In: Kibrick, R.I., Wallander, A. (Eds.), Society of Photo-Optical Instrumentation Engineers (SPIE) Conference Series. Society of Photo-Optical Instrumentation Engineers (SPIE) Conference Series, vol. 4011, pp. 2-10.

Berczik, P., Merritt, D., Spurzem, R., 2005. Long-term evolution of massive black hole binaries. II. Binary evolution in low-density galaxies. APJ 633 (November), 680.

Berczik, P., Merritt, D., Spurzem, R., Bischof, H.-P., 2006. Efficient merger of binary supermassive black holes in nonaxisymmetric galaxies. APJL 642 (May), L21.

Berentzen, I., Preto, M., Berczik, P., Merritt, D., Spurzem, R., 2009. Binary black hole merger in galactic nuclei: post-Newtonian simulations. Astrophys. J. 685 (April), 455.

Elstner, D., Korhonen, H., 2005. Flip-flop phenomenon: observations and theory. Astronomische Nachrichten 326 (April), 278.

Foster, I.T., 2008. Service oriented computing. In: ICSOC 2008. LNCS 5364 5364/ $2008,3 \mathrm{ff}$.

Fukushige, T., Makino, J., Kawai, A., 2005. GRAPE-6A: A single-card GRAPE-6 for parallel PC-GRAPE cluster systems. PASJ 57 (December), 1009.

Harfst, S., Gualandris, A., Merritt, D., Spurzem, R., Portegies Zwart, S., Berczik, P., 2007. Performance analysis of direct N-body algorithms on special-purpose supercomputers. New Astron. 12 (July), 357.

Hessman, F.V., 2006. Remote telescope markup language (RTML). Astronomische Nachrichten 327 (September), 751

Högqvist, M., Röblitz, T., Reinefeld, A., 2007. Stellaris: An RDF-based Information service for AstroGrid-D. In: German e-Science Conference, Baden-Baden, Germany.

Hurley, J.R., Aarseth, S.J., Shara, M.M., 2007. The core binary fractions of star clusters from realistic simulations. APJ 665 (August), 707.

Kuntschke, R., Kemper, A., 2006a. Data stream sharing. In: Current Trends in Database Technology - EDBT 2006, EDBT 2006 Workshop PhD, DataX, IIDB, IIHA, ICSNW, QLQP, PIM, PaRMa, and Reactivity on the Web, Munich, Germany, March 26-31, 2006, Revised Selected Papers. Lecture Notes in Computer Science (LNCS), vol. 4254. Springer Verlag, pp. 769-788.

Kuntschke, R., Kemper, A., 2006b. Matching and evaluation of disjunctive predicates for data stream sharing. In: Proceedings of the ACM International Conference on Information and Knowledge Management (CIKM), Arlington, VA, USA, pp. 832 833.

Kuntschke, R., Stegmaier, B., Kemper, A., Reiser, A., 2005. Streamglobe: Processing and sharing data streams in grid-based p2p infrastructures. In: Proceedings of the International Conference on Very Large Data Bases (demo), Trondheim, Norway, pp. 1259-1262.

Kuntschke, R., Scholl, T., Huber, S., Kemper, A., Reiser, A., Adorf, H.-M., Lemson, G., Voges, W., 2006. Grid-based data stream processing in e-Science. In: Proceedings of the IEEE International Conference on e-Science and Grid Computing. Amsterdam, The Netherlands, p. 30.

Makino, J., Fukushige, T., Koga, M., Namura, K., 2003. GRAPE-6: Massively-parallel special-purpose computer for astrophysical particle simulations. PASJ 55 (December), 1163

Reinecke, M., Dolag, K., Hell, R., Bartelmann, M., Enßlin, T.A., 2006. A simulation pipeline for the Planck mission. AA 445 (January), 373.

Scholl, T., Bauer, B., Gufler, B., Kuntschke, R., Weber, D., Reiser, A., Kemper, A., 2007a. HiSbase: Histogram-based P2P main memory data management. In: Proceedings of the International Conference on Very Large Data Bases (demo), Vienna, Austria, pp. 1394-1397.

Scholl, T., Kuntschke, R., Reiser, A., Kemper, A., 2007b. Community training Partitioning schemes in good shape for federated data grids. In: Proceedings of the IEEE International Conference on e-Science and Grid Computing, Bangalore, India, pp. 195-203.

Scholl, T., Bauer, B., Gufler, B., Kuntschke, R., Reiser, A., Kemper, A., 2009a. Scalable community-driven data sharing in e-science grids. Future Gener. Comput. Syst. 25 (3), 290 <http://dx.doi.org/10.1016/j.future.2008.05.006>.

Scholl, T., Bauer, B., Müller, J., Gufler, B., Reiser, A., Kemper, A., (2009b) Workloadaware data partitioning in community-driven data grids. In: Proceedings of the International Conference on Extending Database Technology (EDBT), SaintPetersburg, Russia, pp. 36-47.

Springel, V., 2005. The cosmological simulation code GADGET-2. MNRAS 364 (December), 1105.

Springel, V., White, S.D.M., Jenkins, A., Frenk, C.S., Yoshida, N., Gao, L., Navarro, J. Thacker, R., Croton, D., Helly, J., Peacock, J.A, Cole, S., Thomas, P. Couchman, H. Evrard, A., Colberg, J., Pearce, F., 2005. Simulations of the formation, evolution and clustering of galaxies and quasars. Nature 435 (Jun.), 629.

Spurzem, R., 1999. Direct N-body Simulations. J. Comput. Appl. Math. 109 (September), 407.

Spurzem, R., Berczik, P., Berentzen, I., Merritt, D., Nakasato, N., Adorf, H.M. Brüsemeister, T., Schwekendiek, P., Steinacker, J., Wambsganß, J., Martinez, G.M., Lienhart, G., Kugel, A., Männer, R., Burkert, A., Naab, T., Vasquez, H., Wetzstein, M., 2007. From Newton to Einstein N-body dynamics in galactic nuclei and SPH using new special hardware and astrogrid-D. J. Phys. Conf. Ser. 78 (1), 012071.

Spurzem, R., Berentzen, I., Berczik, P., Merritt, D., Amaro-Seoane, P., Harfst, S., Gualandris, A., 2008. Parallelization, special hardware and post-Newtonian 
dynamics in direct N-body simulations. In: Aarseth, S.J., Tout, C.A., Mardling, R.A. (Eds.), Lecture Notes in Physics, vol. 760. Springer Verlag, Berlin, p. 377. Strassmeier, K.G., Granzer, T., Weber, M., Woche, M., Andersen, M.I., Bartus, J., Bauer, S.-M., Dionies, F., Popow, E., Fechner, T., Hildebrandt, G., Washuettl, A., Ritter, A., Schwope, A., Staude, A., Paschke, J., Stolz, P.A., Serre-Ricart, M., de la Rosa, T.,
Arnay, . The STELLA robotic observatory. Astronomische Nachrichten 325 (October), 527.

WebLinks, 2010. <http://www.astrogrid-d.org/project-documents/Posters/ publications/citations-na.html>. 